\title{
Identification of microRNAs and their Endonucleolytic Cleavaged target mRNAs in colorectal cancer
}

Fangbin Zhou ${ }^{1,2}$, Donge Tang ${ }^{1}$, Yong Xu ${ }^{1}$, Huiyan $\mathrm{He}^{1}$, Yan Wu${ }^{1}$, Liewen Lin ${ }^{1}$, Jun Dong ${ }^{3}$, Wenyong Tan ${ }^{1,4^{*}}$ and Yong Dai ${ }^{1 *}$

\begin{abstract}
Background: Colorectal cancer (CRC) ranks the third among the most common malignancies globally. It is well known that microRNAs (miRNAs) play vital roles in destabilizing mRNAs and repressing their translations in this disease. However, the mechanism of miRNA-induced mRNA cleavage remains to be investigated.
\end{abstract}

Method: In this study, high-throughput small RNA (sRNA) sequencing was utilized to identify and profile miRNAs from six pairs of colorectal cancer tissues (CTs) and adjacent tissues (CNs). Degradome sequencing (DS) was employed to detect the cleaved target genes. The Database for Annotation, Visualization and Integrated Discovery (DAVID) software was used for GO (Gene Ontology) and KEGG (Kyoto Encyclopedia of Genes and Genomes) pathway analysis.

Results: In total, 1278 known miRNAs (clustered into 337 families) and 131 novel miRNAs were characterized in the CT and CN libraries, respectively. Of those, 420 known and eight novel miRNAs were defined as differentially expressed miRNAs (DEmiRNAs) by comparing the expression levels observed in the CT and CN libraries. Furthermore, through DS, 9685 and 202 potential target transcripts were characterized as target genes for 268 known and 33 novel miRNAs, respectively. It was further predicted that a total of 264 targeted genes for the 85 DEmiRNAs are involved in proteoglycans in cancer and the AMP-activated protein kinase signaling pathway. After systemic analysis of prognosisrelated miRNA targets in those cancer-related signal pathways, we found that two targets ezrin (EZR) and hematopoietic cell-specific Lyn substrate 1 (HCLS1) had the potential prognostic characteristics with CRC regarding over survival (OS) or recurrence.

Conclusion: In total, we found that endonucleolytic miRNA-directed mRNA cleavage occurs in CRC. A number of potential genes targeted by CRC-related miRNAs were identified and some may have the potential as prognosis markers of CRC. The present findings may lead to an improved better appreciation of the novel interaction mode between miRNAs and target genes in CRC.

Keywords: microRNA, Colorectal Cancer, High-throughput small RNA sequencing, Degradome sequencing, Endonucleolytic cleavage

\footnotetext{
* Correspondence: tanwyym@hotmail.com; daiyong22@aliyun.com

${ }^{1}$ Clinical Medical Research Center, The Second Clinical Medical College of

Jinan University (Shenzhen People's Hospital), 1017 North Rd Dongmen,

Luohu District, Shenzhen, China

Full list of author information is available at the end of the article
}

(C) The Author(s). 2020 Open Access This article is licensed under a Creative Commons Attribution 4.0 International License, which permits use, sharing, adaptation, distribution and reproduction in any medium or format, as long as you give appropriate credit to the original author(s) and the source, provide a link to the Creative Commons licence, and indicate if changes were made. The images or other third party material in this article are included in the article's Creative Commons licence, unless indicated otherwise in a credit line to the material. If material is not included in the article's Creative Commons licence and your intended use is not permitted by statutory regulation or exceeds the permitted use, you will need to obtain permission directly from the copyright holder. To view a copy of this licence, visit http://creativecommons.org/licenses/by/4.0/. The Creative Commons Public Domain Dedication waiver (http://creativecommons.org/publicdomain/zero/1.0/) applies to the data made available in this article, unless otherwise stated in a credit line to the data. 


\section{Background}

MicroRNAs (miRNAs) represent a group of small non-coding RNAs with approximately 22 nucleotide-long, repressing the expression of complementary mRNA targets. Since first found by Lee et al. [1], miRNAs have been intensively investigated. Numerous researches have demonstrated the regulatory roles of miRNAs in tumorigenesis and development.

miRNAs inhibit the post-transcription of target mRNAs by destabilizing or degrading them. However, the mechanism of miRNAs and their target mRNA interactions between plants and animals are generally different. In plants, the endonucleolytic cleavage guided by miRNAs, rather than translational repression, decided target mRNAs' fate [2]. Most miRNAs base pair to cleaved target mRNAs with high complementarity, which was further degraded by RNA-induced silencing complex (RISC). Numerous studies have demonstrated this plantspecific phenomenon [3-5]. However, in animals (including human), destabilization or translational repression of target mRNAs are established miRNA-targeting pathways via miRNA binding to partially complementary sites $[6,7]$. Cleavage of miRNA targets is relatively rare, and only a few miRNAs exhibit this "superpower" in animals [8-12]. HOXB8 mRNA was the first reported miRNA-directed cleavage target in mouse embryos. miR-196 represses Hoxb8 expression by direct cleavage of complementary region with Hoxb8 [11]. miR-249 bound to the complementary sites of the ZK637.6 transcript to guide the cleavage events in nematodes [12]. As well, mRNA cleavage guided by miRNA was found to mediate the regulation of retrotransposons and viral infection in animal [13-15]. The availability of genesequencing technologies made the comprehensive and systematic analysis of miRNA-induced mRNA cleavage at a whole transcriptome scale possible and degradome sequencing (DS) was then developed. Although originally designed for plants, DS was later successfully applied to animals [16].

In 2012, colorectal cancer (CRC) ranks the third among the most common malignancies globally. It accounted for approximately $10 \%$ of the estimated 14.1 million new cases of cancer [17]. Moreover, it is estimated as the second most deadly type of cancer in both females and males, with 693,600 deaths occurring annually worldwide. The roles of miRNAs in the regulation of CRC (carcinogenesis, development and tumor metastasis), through destabilization or translational repression of target mRNAs, have been widely investigated. However, whether they function by directing cleaving target mRNAs remains to be clarified [18-20]. Thus, herein, we aimed at characterizing the profile of miRNAs and their endonucleolytic cleaved target mRNAs in CRC. Colorectal cancer tissues (CTs) and adjacent tissues (CNs) from six patients with CRC were obtained to construct small RNA (sRNA) libraries for sequencing. DS was further used for the whole transcriptome analysis of miRNA-induced mRNA cleavage in CRC.

\section{Methods}

\section{Study patients}

Samples were surgically resected from CTs (CT3, CT4, CT5, CT6, CT7, CT8) and CNs (CN3, CN4, CN5, CN6, CN7, CN8) of six CRC patients (C3, C4, C5, C6, C7 and C8) without enteritis in Shenzhen People's Hospital (Shenzhen, China), between August and October 2018. The six pairs of samples were immediately frozen and stored in liquid nitrogen. All patients were aged 44-62 years with histologically proven and measurable disease. Of those, C4, C6, and C7 are early-stage I-II patients (CT-E), and C3, C5, and $\mathrm{C} 8$ are advanced-stage III-IV patients (CT-A). Additional, details are listed in Supplementary Table 1.

\section{Preparation of sRNA libraries and sequencing}

Total RNA was extracted from CTs and CNs with the TRIzol reagent (Invitrogen, USA). RNA was qualified and quantified by a NanoDrop 2000 (ThermoFisher, USA). cDNA constructs from the amplification of total RNA were used to prepare sRNA libraries and then sequenced on an Illumina Hiseq2500 at LC-BIO (LC Sciences, China) based on the manufacturer's protocol.

\section{Characterization of known and novel miRNAs}

The in-house software program, ACGT101-miR (LC Sciences, China) was applied to analysis the raw sequencing reads and redundant data such as adaptor dimers were removed according to the software's handbook. Subsequently, known and novel miRNAs were identified by mapping the remaining clean small reads to the miRbase database (Release 22) [21]. The classification criteria for known and novel miRNAs were listed in Supplementary Table 4 . In brief, sequences that perfectly matched were defined as known miRNAs, whereas sequences that were mapped to the human reference genome with no mismatches, but not matched pre-miRNAs of selected species in the miRbase were considered as novel miRNAs.

\section{miRNA validation}

Quantitative RT-PCR (qRT-PCR) was conducted on a qTOWER 2.2 Real-Time PCR System (ANALYTIKJENA, Germany) using the aforementioned RNA samples. First-strand cDNA was synthesized using the TUREscript 1st Strand cDNA SYNTHESIS Kit (Aidlab, China) in $10-\mu$ l reaction volumes containing $0.2 \mathrm{mg}$ total RNA, $2 \mu \mathrm{l} 5 \times \mathrm{RT}$ reaction mix, $0.5 \mu \mathrm{l}$ primers, $0.5 \mu \mathrm{l}$ TUREscript H- RTase/RI Mix and RNase-free dH2O. The reaction procedure was as follow: $25^{\circ} \mathrm{C}$ for $10 \mathrm{~min}$; $42^{\circ} \mathrm{C}$ for $50 \mathrm{~min}$ and $65^{\circ} \mathrm{C}$ for $15 \mathrm{~min}$. A SYBR PrimeScript miRNA RT-PCR Kit (TianGen Biotech, China) was used to carry out qRT-PCR experiments. Each PCR reaction consisted of $1 \mu \mathrm{l} \mathrm{cDNA}$ constructs ( 100 ng), $1 \mu \mathrm{l}$ sequence-specific forward primer, $1 \mu \mathrm{l}$ universal reverse primer, $10 \mu \mathrm{l} 2 \times$ SYBR premix EX Taq II, and $7 \mathrm{ml}$ 
ddH2O. The samples were incubated at $95^{\circ} \mathrm{C}$ for $5 \mathrm{~min}$ followed by 45 cycles at $95^{\circ} \mathrm{C}$ for $15 \mathrm{~s}$ and subsequently $60^{\circ} \mathrm{C}$ for $30 \mathrm{~s}$. The U6 gene was used as an internal control. Each sample performed three independent biological replicates. The primers of miRNAs are listed in Supplementary Table 2.

\section{Degradome analysis and target identification}

Total RNA samples from CTs and CNs were sent to LC$\mathrm{BIO}$ for the preparation of the DS libraries. The experimental steps were as described previously [5]. The Illumina's Cluster Station was unitized for cluster generation of the purified cDNA library and the Illumina Hiseq2500 for sequencing. The Illumina's Pipeline and the CleaveLand 3.0 pipeline software were used to analysis the extracted reads and identify the potentially cleaved targets [22].

\section{Functional analysis of target genes}

Target genes corresponding to different expressed miRNAs (DEmiRNAs) were input into the Database for Annotation, Visualization and Integrated Discovery (DAVID) for gene ontology (GO) and Kyoto Encyclopedia of Genes and Genomes (KEGG) analysis. Fisher's exact test was used for statistical analysis. GO terms and KEGG pathways with a corrected $p \leq 0.05$ were regarded to be significantly enriched.
The correlation between the expression of potential miRNA targets from cancer-related pathways and clinical features were investigated at cBioPortal. The flowchart of the study was presented in Fig. 1.

\section{Results}

High-throughput sequencing of sRNAs for CRC patients Based on the sequencing data obtained from the CTs and CNs of six CRC patients, 12 sRNA libraries were constructed to identify miRNAs. Raw sRNA sequencing reads were deposited at NCBI (https://www.ncbi.nlm. nih.gov/) under accession number GSE125961. 79,012, 674 and 83,084,696 unfiltered raw reads were obtained from the CT and CN libraries, respectively (Supplementary Table 3 ). The analysis yielded a total of 4,262,573 and 2,652,420 unique sequences, respectively. Subsequently, redundant data such as adaptor dimers and junk reads were excluded. Consequently, a total of 1,619, 465 and 824,818 clean sRNA sequence reads remained. The percentages and numbers of different sRNAs are listed in Supplementary Table 3. It was noted that the length interval of unique reads between the $\mathrm{CT}$ and $\mathrm{CN}$ libraries both ranged from 18 to $26 \mathrm{nt}$, with $22 \mathrm{nt}$ being the most predominant (Supplementary Figure 1).

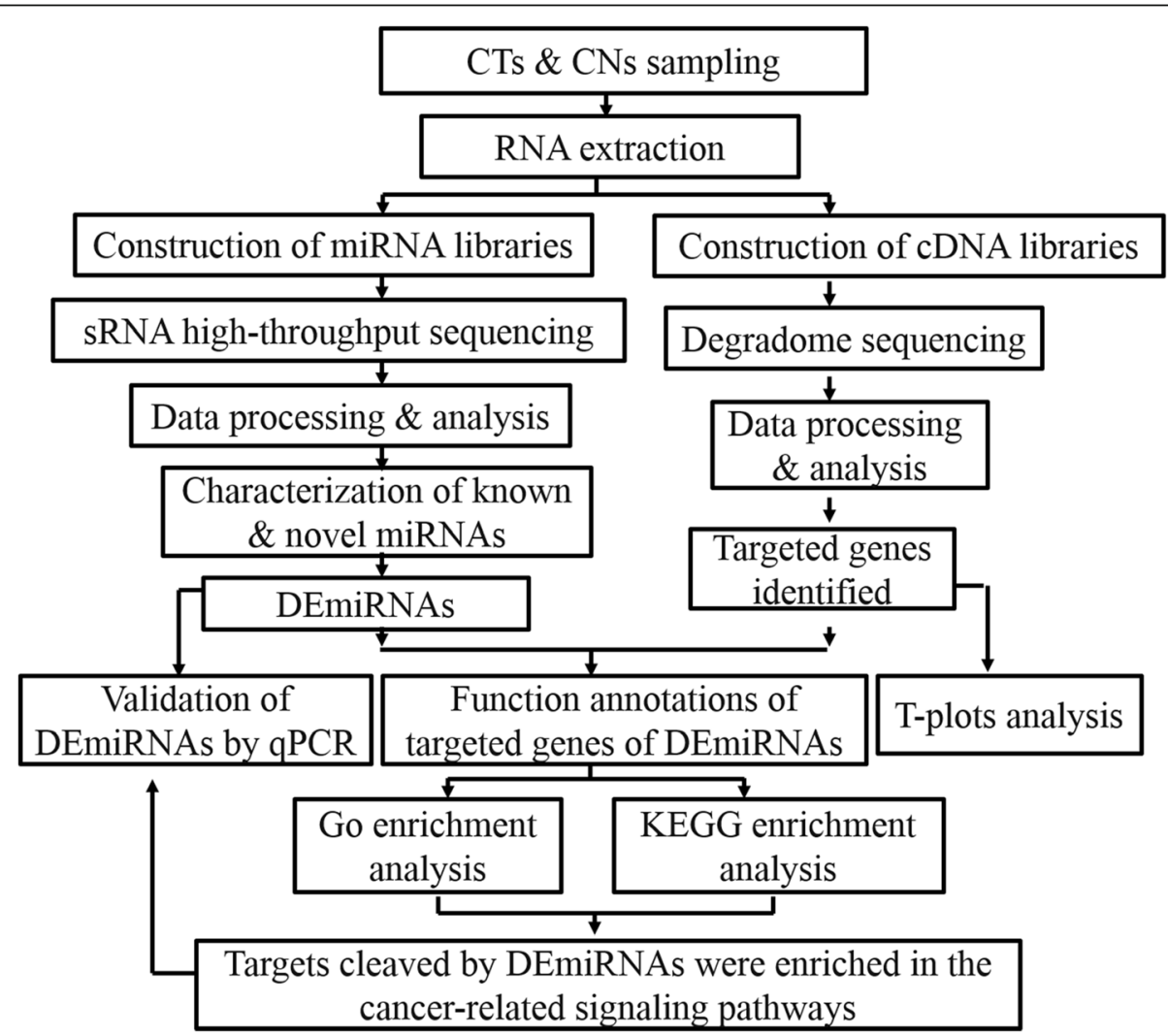

Fig. 1 The flowchart of the study. High-throughput sRNA sequencing was employed to identify and profile miRNAs and DS was used to identify the cleaved target genes. After a series of data processing and bioinformatic analysis, targets cleaved by DEmiRNAs were found to be enriched in the cancer-related signaling pathways 


\section{Identification of known and novel miRNAs}

Known miRNAs in CRC were identified by comparing the raw sequencing data with human reference genome and miRBase. Subsequently, 1278 known miRNAs (clustered into 337 families) were characterized in all libraries (Supplementary Table 4). The expression levels of known miRNAs in each library were estimated via the normalization of their original reads as transcripts per million (TPM). An extremely variable frequency spectrums of miRNA were observed, ranging from 0 to $>1,000,000 \mathrm{TPM}$. The most abundant miRNAs in the $\mathrm{CT}$ and $\mathrm{CN}$ libraries were hsamiR-143-3p_R + 1 and hsa-miR-143-3p_R +1 , respectively. In contrast, hsa-miR-381-5p_R +1 and hsa-miR383-5p showed low expression levels.

After removing known miRNAs, clean unique reads were employed for the identification of novel miRNAs in CRC. Ultimately, 131 novel miRNAs were detected in the 12 libraries (Supplementary Table 5). It was noted that the expression of most novel miRNAs identified exhibited relatively low, which was consistent with the results of previous researches in other human models [9].

\section{Differential expression analysis of known and novel miRNAs between $\mathrm{CT}$ and $\mathrm{CN}$ libraries}

In total, 428 differentially expressed miRNAs (DEmiRNAs) between the $\mathrm{CT}$ and $\mathrm{CN}$ libraries were identified (293 and 135 were upregulated and downregulated, respectively) (Fig. 2). In detail, 420 of the 1090 known DEmiRNAs were detected in the libraries (Supplementary Table 6). Of these, 64, 171, and 185 were miRNAs yielded $p$ value $\leq 0.001$, $\leq$ 0.01 , and $\leq 0.05$, respectively. In detail, 51 miRNAs were up-regulated, whereas 13 were down-regulated $(p \leq 0.001)$; 109 miRNAs were up-regulated, whereas 62 were downregulated $(p \leq 0.01) ; 125$ miRNAs were up-regulated, whereas 60 were down-regulated $(p<=0.05)$. Notably, hsamiR-548au-5p_R-1 was the most significantly up-regulated miRNA with a $p$-value of 2.09E-10, whereas hsa-miR-1271$5 \mathrm{p}$ was the most significantly down-regulated miRNA with a $p$-value of $1.56 \mathrm{E}-04$.

Eighty-one novel miRNAs were identified in the CT and $\mathrm{CN}$ libraries, the vast majority of which were expressed at a relatively low level (Supplementary Table 7). Only eight were differentially expressed and all of them were upregulated, of which, PC-3p-30435_41 was the most significantly with a $p$-value as 7.77E-09 (Table 1).

DEmiRNAs were also characterized between the earlystage I-II (CT-E) and advanced-stage III-IV (CT-A) libraries to detect the CRC pathological grading-related miRNAs. However, only six DEmiRNAs were identified, of which, hsa-miR-10,527-5p, cgr-miR-1260_L + 1, hsamiR-301b-5p, and dno-miR-450c-5p, hsa-miR-548az-5p_ $\mathrm{L}-1 \mathrm{R}+1$ were upregulated, and only hsa-miR-874-3p_ $R+1$ was downregulated (Supplementary Table 8). None of any novel miRNAs belonged to DEmiRNAs.

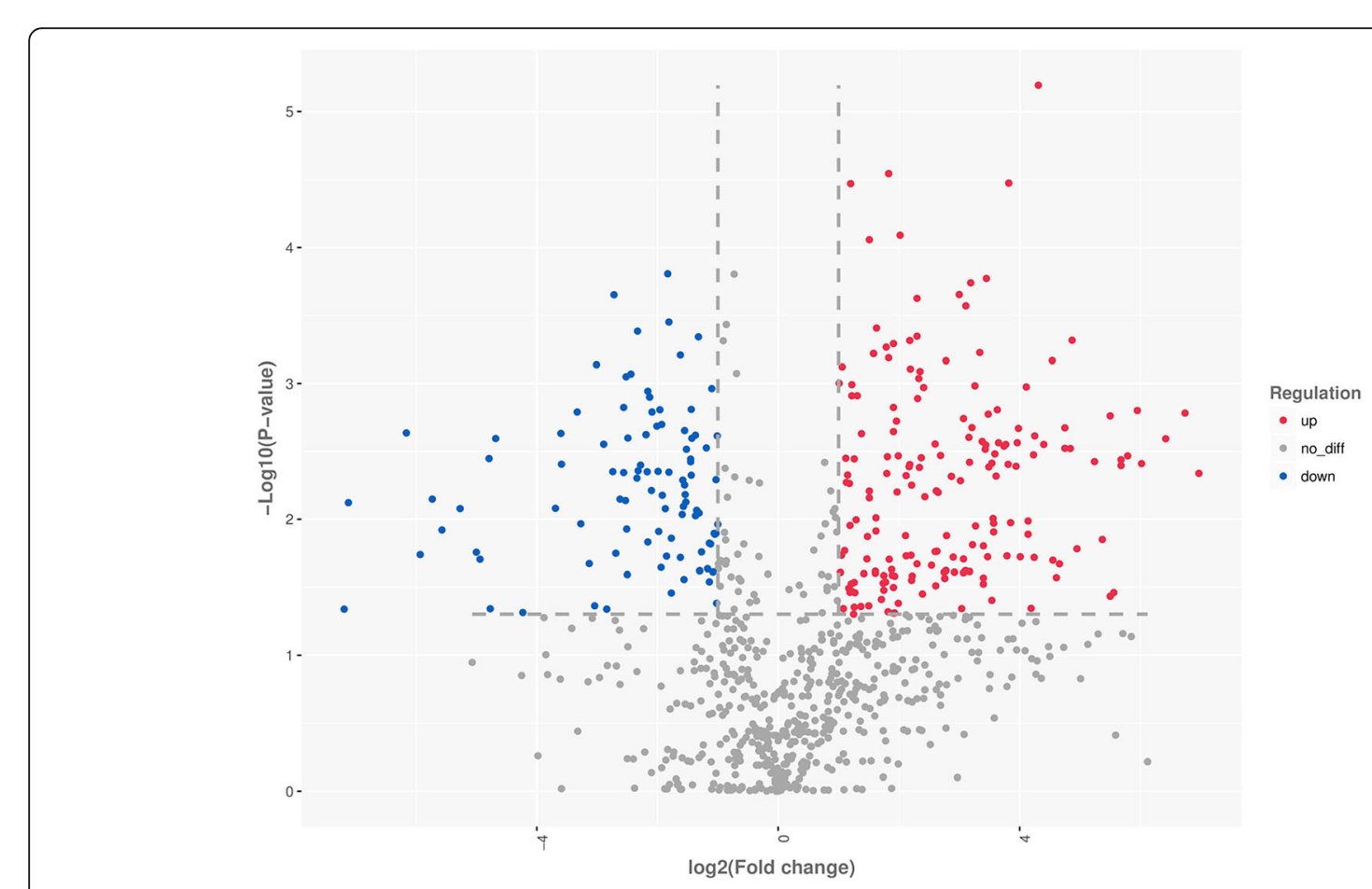

Fig. 2 Volcanic diagrams showing the number of DEmiRNAs between CTs and CNs. The red dots indicate miRNAs with significant differences, and the blue dots indicate that miRNAs without significant differences 
Table 1 The top 10 significantly differentially expressed novel miRNAs between the CT and CN libraries

\begin{tabular}{|c|c|c|c|c|c|}
\hline miR_name & miR_seq & Up/down & $\mathrm{FC}$ & $\log 2(\mathrm{Fc})$ & $\boldsymbol{P}$ value \\
\hline PC-3p-30435_41 & CTACCCCAGGATGCCAGCATAGTT & up & inf & inf & 7.77E-09* \\
\hline PC-3p-24565_58 & ATGTTATGATGATGGGCGAAA & up & inf & $\inf$ & $9.85 \mathrm{E}-08^{*}$ \\
\hline PC-5p-23775_61 & TAGGGTGATGAAAAAGAAT & up & 10.40 & 3.38 & 2.67E-03* \\
\hline PC-5p-34972_32 & CACCCTITCCTGTGCCCTIT & up & inf & $\inf$ & $4.24 \mathrm{E}-03^{*}$ \\
\hline PC-5p-16080_103 & TTAGTGGCTCCCTCTGCCTGCA & up & 8.12 & 3.02 & $5.20 \mathrm{E}-03^{*}$ \\
\hline PC-5p-2366_1399 & TTGCAAGCAACACTCTGTGGCAGA & up & 6.19 & 2.63 & $1.72 \mathrm{E}-02^{*}$ \\
\hline PC-5p-37743_28 & GTGGAGGACTGAGAAGGTGAGGC & up & 3.68 & 1.88 & $2.34 \mathrm{E}-02^{*}$ \\
\hline PC-5p-31711_38 & AATAATGATGATGACATACTGATA & up & inf & $\inf$ & $2.52 \mathrm{E}-02^{*}$ \\
\hline PC-5p-22018_68 & ATTCCCACTGTCCCTACCTAT & up & 6.19 & 2.63 & $6.67 \mathrm{E}-02$ \\
\hline PC-5p-77988_5 & TCAGACACAGGTATGGCTGGCTCT & up & $\inf$ & $\inf$ & 7.56E-02 \\
\hline
\end{tabular}

Inf Infinity, FC Fold change; *, $P \leq 0.05$

\section{Validation of DEmiRNAs by qRT-PCR}

The expression of seven randomly selected miRNAs was validated using $\mathrm{qRT}$-PCR to verify the high-throughput sequencing results. qPCR analysis indicated that, although the fold change of expression did not completely coincide with that measured using the sequencing data, the trend performed similarity (Fig. 3). For example, both the sequencing and qRT-PCR results confirmed the up-regulation of hsa-miR-106b-5p, hsa-mir-148a-3p and hsa-miR-196a-5p in the CT libraries compared with CN libraries, and the down-regulation of hsa-miR-145-5p in the CT libraries. These results indicated the reliability of high-throughput sequencing as a method to predict the expression of miNRAs.

\section{Identification of target genes of miRNAs using DS}

Target genes of miRNAs in CRC were identified by DS at a global level and the raw sequencing data is uploaded at NCBI with accession number GSE125962. 29,604,631 raw reads were acquired from the libraries. Following the removal of the reads without the CAGCAG adaptor, 4,855, 908 unique raw reads remained, which were subsequently mapped to the human genome database. 22,187,626 transcript-mapped reads were obtained and the mapped reads represented annotated human genes in the library.

A potential cleaved target was defined based on the algorithm of the CleaveLand pipeline. In total, 9685 transcripts containing 2797 target genes were characterized as targets for 268 known miRNAs. Of those, 110, 112, 2261, 423, and 6779 were categorized into subgroups 0 , $1,2,3$, or 4 in the libraries (Supplementary Table 9). The T-plots for some targets are illustrated in Fig. 4. Among the 268 known miRNAs, some target only one transcript (eg, bta-miR-12034_1ss19TG), whereas the others have multiple targets (bta-miR-11987_L-1_ 1 ss8TA). In addition, we noticed that the majority of targets were anchored in protein coding locus, which had been previously reported in certain plants [3]. Of the
9685 transcripts, only 358 (3.70\%) target sites located in untranslated region (Table 2). Functional analysis indicated that these target genes were concentrated in mRNA transport, endocytosis, early endosome to late endosome transport, protein phosphorylation, etc.

For novel miRNAs, 200 transcripts covering 47 target genes were identified as targets for 33 novel miRNAs. Of those, $1,0,64,3$, and 132 transcripts were categorized into subgroups $0,1,2,3$, or 4 in the libraries (Table 2, Supplementary Table 10). Most of these novel miRNAs belonged to categories 2 and 4. Similarly, only 7 (3.50\%) target sites located in untranslated region. And among the 33 novel miRNAs, some have only one targeted transcript, whereas the others were polygamous. Among the latter group of miRNAs, PC-3p-67706_6 exhibited the highest number of targets (20 transcripts). Both the high-throughput sequencing and qPCR results confirmed that the levels of PC-3p-67706_6 was upregulated in the $\mathrm{CT}$ libraries compared with $\mathrm{CN}$ libraries (Fig. 5). Functional descriptions of these target genes were also conducted; however, fewer functions were detected using the $\mathrm{GO}$ analysis.

For six DEmiRNAs between CT-E and CT-A, we found that only the miRNA cgr-miR-1260_L +1 had nine transcripts covering three target genes.

\section{Function analysis of the potential miRNA targets}

Among the known miRNAs, 263 target genes for 84 of the 420 DEmiRNAs were validated using DS. However, for novel miRNAs, only one DEmiRNA (PC-5p-23775 61), was identified to direct cleaver the target gene. The whole of 264 target genes for these 84 DEmiRNAs were subjected to Fisher's exact test-based GO biological and functional analysis to investigate the processes involved in CRC development and progression (Supplementary Table 11). The results of the functional analysis of miRNAs were generated using DAVID. According to number of genes involved, we listed the top eight significantly 


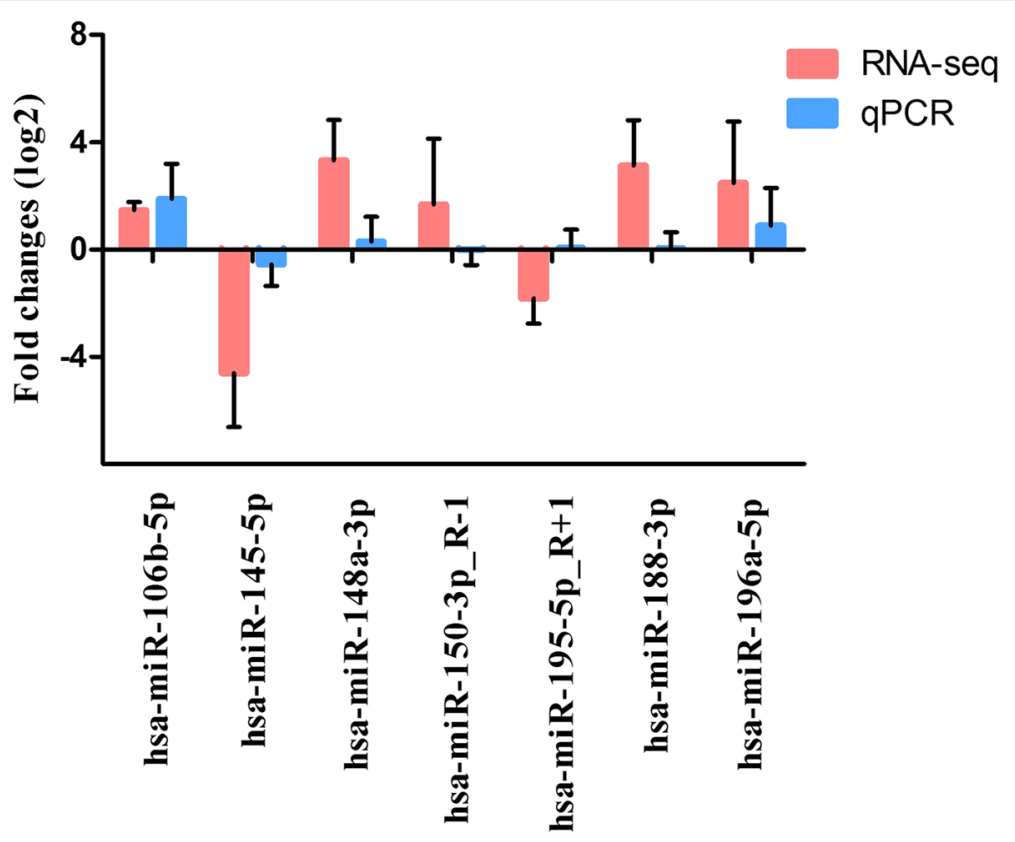

Fig. 3 Comparisons of the expression levels of seven miRNAs between RNA-seq and qPCR. Fold change (log2) in CN libraries relative to CT libraries were detected by RNA-seq. The relative expression levels of miRNAs were normalized to the expression of U6 in qPCR

related GO terms in Table 3. The results indicated that in the biological process (BP), the targeted genes were mainly enriched in membrane protein proteolysis, regulation of nucleic acid-templated transcription, mRNA processing, negative regulation of non-canonical Wnt signaling pathway, negative regulation of transforming growth factor beta receptor (TGF- $\beta$ ) signaling pathway, negative regulation of TOR signaling, etc. In the cellular component (CC) analysis, the genes were concentrated in nucleoplasm, lysosomal membrane, membrane, nuclear membrane and spindle pole centrosome. For molecular function (MF), the upregulated genes were mainly anchored in protein binding, poly(A) RNA binding, ATPase activity, actin binding, enzyme binding, etc. The results implied biological functions of miRNAs in the regulation of genes of CRC.

A KEGG pathway analysis was conducted to recognize the signaling pathways involved in the progression of CRC. The eight KEGG pathways identified in our study are listed in Table 4. Among these, the proteoglycans in cancer and AMPK signaling pathways were cancerrelated. Actin gamma 1 (ACTG1), DEAD-box helicase 5 (DDX5), ezrin (EZR), fibronectin 1 (FN1), hematopoietic cell-specific Lyn substrate 1 (HCLS1), phosphoinositide3-kinase regulatory subunit 5 (PIK3R5) and SOS Ras/ Rho guanine nucleotide exchange factor 2 (SOS2) were the potential miRNA targets in proteoglycans in cancer, AKT1 substrate 1 (AKT1S1), cystic fibrosis transmembrane conductance regulator (CFTR), PIK3R5, protein kinase AMP-activated non-catalytic subunit beta 1 (PRKAB1) and protein phosphatase 2 regulatory subunit
Bgamma (PPP2R2C) were the potential miRNA targets in the AMPK signaling pathway, and actin gamma 1 (ACTG1) and symplekin (SYMPK) were part of the potential miRNA targets in the tight junction pathway. Also, we verified the expression of these 11 miRNA targeted genes using qRT-PCR (Fig. 5). Both the highthroughput sequencing and qRT-PCR results confirmed that the levels of bta-miR-1246_L + 1R-2, mmu-miR3968_L-3_1ss14AT, hsa-miR-665_R-2, hsa-miR-1268a, hsa-miR-548ae-5p and hsa-mir-7110-p3_1ss18AC were up-regulated in the CT libraries compared with $\mathrm{CN}$ libraries, whereas that of mmu-mir-6236-p3_2, hsa-miR383-5p and hsa-miR-197-3p was down-regulated in the CT libraries. The above results further validated the reliability of the high-throughput sequencing as a method to predict the expression of miNRAs.

Non-cancer related pathways, such as tight junction, amoebiasis, focal adhesion, regulation of actin cytoskeleton, and mRNA surveillance pathway were also enriched. Examples of targeted genes involved into proteoglycans in cancer pathway and the corresponding miRNAs were exhibited in Fig. 6. These results exerted that miRNAs played a vital role in the regulation of CRC initiation, progression, and metastasis by affecting the proteoglycans in cancer pathway.

Clinical significance of miRNA targets in proteoglycans in cancer and the AMPK signaling pathways

To identify potential targeted genes with prognostic characteristics, the CRC dataset of TCGA with larger 


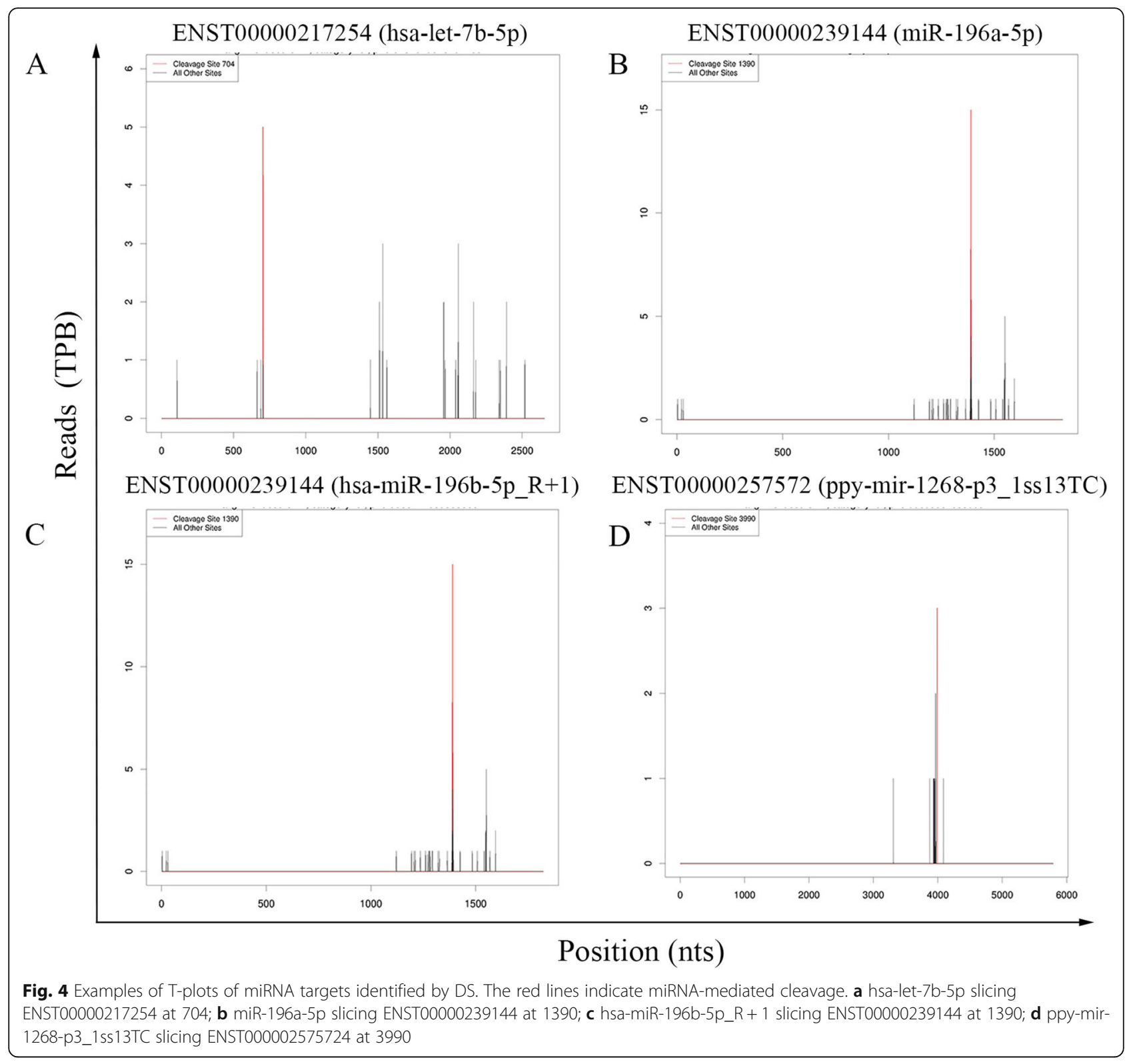

382 tissue samples were interrogated to profile the mRNA expression levels of targeted genes using survival Kaplan-Meier estimate at cBioPortal. As a result, using Onco Query Language (OQL) EXP > 2, in proteoglycans in cancer signaling pathway, two targets EZR and HCLS1 were found to be negatively correlated with overall survival (OS) $(P=4.94 \mathrm{E}-4$ and 0.011$)$ (Fig. 7a). However, none of targets were found be significantly associated with OS in the AMPactivated protein kinase signaling pathway (Fig. 7b). Recurrence is a major concern for cancer survivors. The results indicated that EZR was negatively correlated with progressionfree survival (PFS) in proteoglycans in cancer signaling pathway $(P=0.032)$ (Fig. 8a). On the contrary, none of targets were found be significantly associated with PFS in the AMPactivated protein kinase signaling pathway (Fig. 8b).
To reveal the clinical significance of these 11 miRNA targets, mRNA expression alterations, as well as alterations to other clinical features, such as sex, age in diagnosis, tumor stage, histologic type, metastatic status and KRAS mutation, were investigated. However, apart from FN1 being identified to be associated with diagnosis age $(P=0.021)$ (Fig. 9), other clinical features, including sex, tumor stage, histologic type, metastatic status and KRAS mutation, were evaluated separately, and no significant associations with 11 miRNA targets were observed (Supplementary Figs. 2, 3 and 4).

\section{Discussion}

sRNAs, including miRNAs, affect the post-transcription of target genes via multiple mechanisms, including 
Table 2 The locations of transcripts targeted by known and novel miRNAs

\begin{tabular}{|c|c|c|c|c|}
\hline miRNAs & Degradome categories & Protein coding regions ( $\mathrm{N} / \%)$ & Untranslated regions ( $\mathrm{N} / \%$ ) & $\begin{array}{l}\text { Total } \\
(\mathrm{N})\end{array}$ \\
\hline \multirow[t]{6}{*}{ Known } & 0 & $106(96.36 \%)$ & $4(3.64 \%)$ & 110 \\
\hline & 1 & $103(91.96 \%)$ & $9(8.04 \%)$ & 112 \\
\hline & 2 & 2159 (95.49\%) & $102(4.51 \%)$ & 2261 \\
\hline & 3 & $413(97.64 \%)$ & $10(2.36 \%)$ & 423 \\
\hline & 4 & $6546(96.56 \%)$ & $233(3.44 \%)$ & 6779 \\
\hline & total & $9327(96.30 \%)$ & $358(3.70 \%)$ & 9685 \\
\hline \multirow[t]{6}{*}{ Novel } & 0 & $1(100.00 \%)$ & $0(0.00 \%)$ & 1 \\
\hline & 1 & $0(N A)$ & 0 (NA) & 0 \\
\hline & 2 & $63(98.44 \%)$ & $1(1.56 \%)$ & 64 \\
\hline & 3 & $1(33.33 \%)$ & $2(66.67 \%)$ & 3 \\
\hline & 4 & $128(96.97 \%)$ & $4(3.03 \%)$ & 132 \\
\hline & total & $193(96.50)$ & $7(3.50 \%)$ & 200 \\
\hline
\end{tabular}

NA not applicable

deadenylation or decapping. These functions result in mRNA destabilization by disturbing ribosome recruitment, as well as degradation by direct cleavage of target RNAs [6, 7]. Considering that only Ago2 is characterized in controllable catalytic activity, miRNA-induced mRNA cleavage happens occasionally in animals [11]. The development of DS renders the observation and identification of miRNA-mediated cleavage events more feasible. Jain et al. used DS to reveal 29 cleaved targets of insect miRNAs from malaria host Anopheles stephensi [8].
Ashley et al. found 398 sciRNAs and 810 cleaved target genes in various organs of mouse [13].

In CRC, The involvement of miRNAs in the regulation of gene post-transcription via mRNA translational inhibition or destabilization are well documented $[19,20]$. However, how they function to cleave target mRNAs remain to be elucidated. Herein, we used high-through sequencing and DS technologies to investigate the profile of miRNA-induced mRNA cleavage in CRC. A total of 1278 known and 131 novel miRNAs were identified

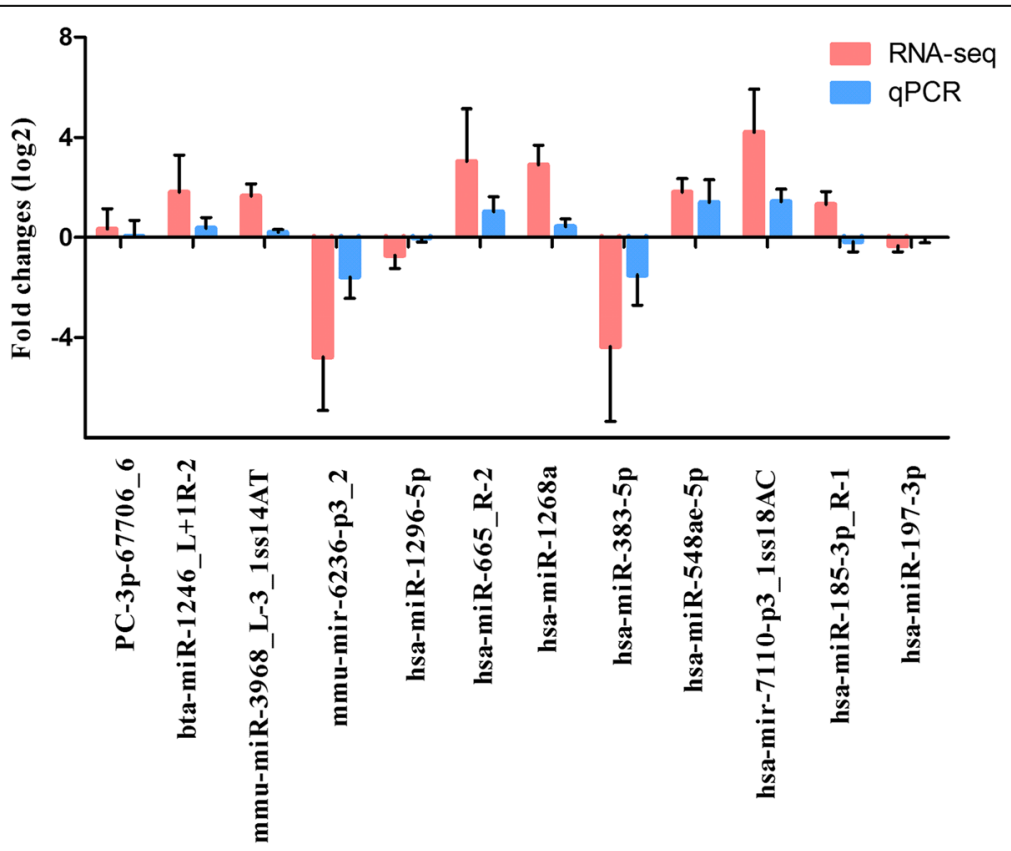

Fig. 5 Comparisons of the expression levels of 12 miRNAs between RNA-seq and qPCR. Fold change (log2) in CN libraries relative to CT libraries were detected by RNA-seq. The relative expression levels of miRNAs were normalized to the expression of U6 in qPCR 
Table 3 Go analysis of mRNAs targets of DEmiRNAs

\begin{tabular}{|c|c|c|}
\hline Term & Count & $\boldsymbol{P}$ Value $^{\mathbf{a}}$ \\
\hline \multicolumn{3}{|l|}{ GO-BPs } \\
\hline GO:0033619 membrane protein proteolysis & 3 & 0.005678 \\
\hline GO:1903506 regulation of nucleic acid-templated transcription & 3 & 0.014466 \\
\hline GO:0006397 mRNA processing & 7 & 0.018271 \\
\hline GO:2000051 negative regulation of non-canonical Wnt signaling pathway & 2 & 0.034437 \\
\hline GO:0030512 negative regulation of transforming growth factor beta receptor signaling pathway & 4 & 0.038329 \\
\hline GO:0032007 negative regulation of TOR signaling & 3 & 0.04162 \\
\hline GO:0008380 RNA splicing & 6 & 0.044499 \\
\hline GO:0000398 mRNA splicing, via spliceosome & 7 & 0.045339 \\
\hline \multicolumn{3}{|l|}{ GO-CCs } \\
\hline GO:0005654 nucleoplasm & 57 & 8.50E-06 \\
\hline GO:0005765 lysosomal membrane & 11 & 0.001198 \\
\hline GO:0016020 membrane & 41 & 0.001693 \\
\hline GO:0031965 nuclear membrane & 9 & 0.004743 \\
\hline GO:0031616 spindle pole centrosome & 3 & 0.005441 \\
\hline GO:0000118 histone deacetylase complex & 4 & 0.007827 \\
\hline GO:0005925 focal adhesion & 11 & 0.014141 \\
\hline GO:0030660 Golgi-associated vesicle membrane & 3 & 0.023398 \\
\hline \multicolumn{3}{|l|}{ GO-MFs } \\
\hline GO:0005515 protein binding & 134 & 4.51E-05 \\
\hline GO:0044822 poly(A) RNA binding & 30 & 7.93E-05 \\
\hline GO:0016887 ATPase activity & 9 & 0.00165 \\
\hline GO:0003779 actin binding & 11 & 0.001948 \\
\hline GO:0019899 enzyme binding & 10 & 0.018955 \\
\hline GO:0005102 receptor binding & 10 & 0.026515 \\
\hline GO:0003743 translation initiation factor activity & 4 & 0.036608 \\
\hline GO:0004583 dolichyl-phosphate-glucose-glycolipid alpha-glucosyltransferase activity & 2 & 0.047016 \\
\hline
\end{tabular}

${ }^{a} B P$ biological process, $M F$ molecular function, $C C$ cellular component. $P$ value $<0.05$ was considered as threshold values of significant difference

Table 4 KEGG pathway analysis of mRNAs targets of DEmiRNAs

\begin{tabular}{lll}
\hline KEGG pathways & Count & $\boldsymbol{P}$ Value \\
\hline hsa04530:Tight junction & 6 & 0.006847 \\
hsa05146:Amoebiasis & 6 & 0.015314 \\
hsa04510:Focal adhesion & 8 & 0.023369 \\
hsa04810:Regulation of actin cytoskeleton & 8 & 0.025633 \\
hsa03015:mRNA surveillance pathway & 5 & 0.036679 \\
hsa05205:Proteoglycans in cancer & 7 & 0.047755 \\
hsa04670:Leukocyte transendothelial migration & 5 & 0.074216 \\
hsa04152:AMPK signaling pathway & 5 & 0.089858 \\
\hline
\end{tabular}

$P$ value $<0.05$ was considered as threshold values of significant difference from the $\mathrm{CT}$ and $\mathrm{CN}$ libraries, respectively. Certain established miRNAs involved in vital signaling pathways, such as miR-1271 and miR-490-3p, were identified. Wnt signals play important roles in the regulation of cancer cell proliferation and migration. Sun et al. reported that miRNA-1271 negatively regulated metadherin \& Wnt signaling to inhibit cell proliferation and invasion of CRC [23]. Zheng et al. indicated that miR-490-3p worked as a suppressor to mediate CRC progression via the control of the Wnt/ $\beta$-catenin signaling pathway [24]. Moreover, miR-17, miR-21 and miR-106a have been identified to regulate TGF- $\beta$ receptor 2 , an important member of the TGF- $\beta$ family. In addition, some miRNAs, such as PC-5p-16080_103 and PC-3p-31923_38, were newly identified. Notably, the majority of these novel miRNAs (except PC-3p-3445_793 and PC-5p2366_1399) had relatively low expression (less than 100 TPM), This observation has also reported in certain 


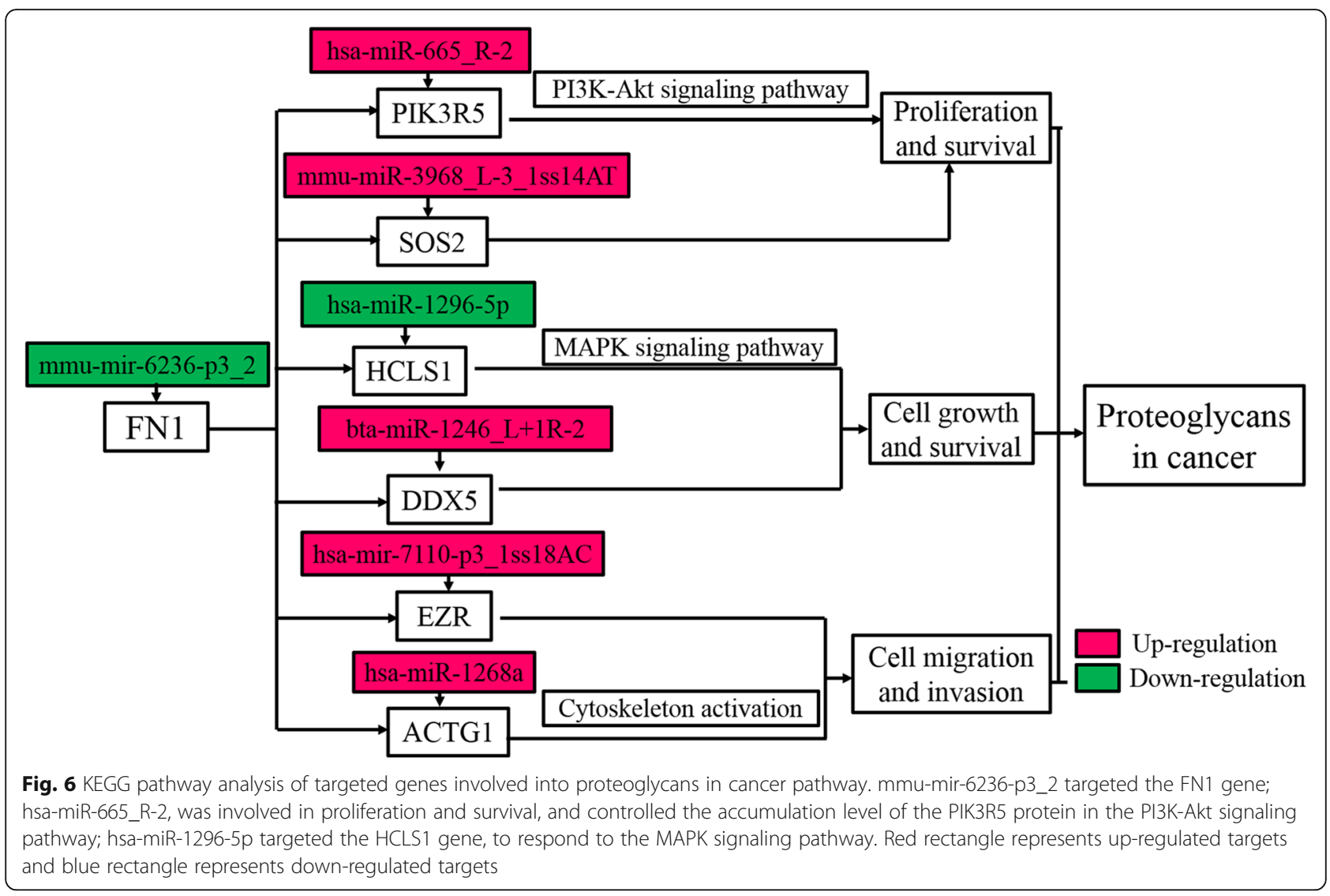

plants $[3,25]$. The above results implied that the expression levels of highly conserved miRNAs was higher than these of less conserved miRNAs. This may partly be attributed to the critical roles of these conserved miRNAs in human evolution. Although most novel miRNAs showed at a low expression levels, their potential roles in CRC carcinogenesis, development, and metastasis remained to be deserved to be investigated.

Four hundred twenty known DEmiRNAs were detected between CT and $\mathrm{CN}$ libraries. Of those, 285 DEmiRNAs were up-regulated. Among the DEmiRNAs, miR-21 is an established miRNA, which is upregulated in CRC and is speculated to be associated with tumor stage and outcome [26]. Notably, 135 known DEmiRNAs were downregulated. Among those, miR-143 was proposed to downgrade in colon cancer and negatively correlated with tumor size and disease-free survival. Moreover, it has the potential for KRAS wild-type CRC prognosis $[27,28]$. The target gene of miR-143 encodes the cysteine and serine rich nuclear protein 3 (CSRNP3), single nucleotide polymorphisms (SNPs) of which has been identified as a novel susceptibility mark for metabolic syndrome [29].

DS provides a powerful tool for the investigation of miRNA-mRNAs interactions at a global level. Herein, a total of 9685 potential target transcripts including 2797 genes were characterized as targets for 268 known miRNAs. The functions of the identified targets of known miRNAs were diverse. For example, hsa-mir-5684-p3 1ss18CG targeting major vault protein (MVP), which was found to be regulated by Notch 1 and contribute to overcome chemoresistance in triple-negative breast cancer cells [30]. hsa-miR-5585-3p_L-3R-1_1ss5AG and hsa-mir-7851-p5_1ss12AC targeting AKT serine/threonine kinase 3 (AKT3) played swinging roles in the tumorigenesis, development, and progression of various types of cancer, including CRC [31-33]. DS identified a total of 202 potential target transcripts, covering 47 target genes for 33 novel miRNAs. Compared with known miRNAs, targets of novel miRNAs were discrete and GO analysis did not identify related functions. These findings implied the young and unstable evolution of these novel miRNAs.

It was noted that DemiRNAs were also identified between the early-stage I-II (CT-E) and advanced-stage IIIIV (CT-A) libraries. However, only six DEmiRNAs were detected and the only miRNA cgr-miR-1260_L + 1 had nine transcripts covering three target genes. The low number of DEmiRNAs identified may be due to the limitation of sample number used and further study are considered to add more cases to objectively draw the profile of miRNA. 


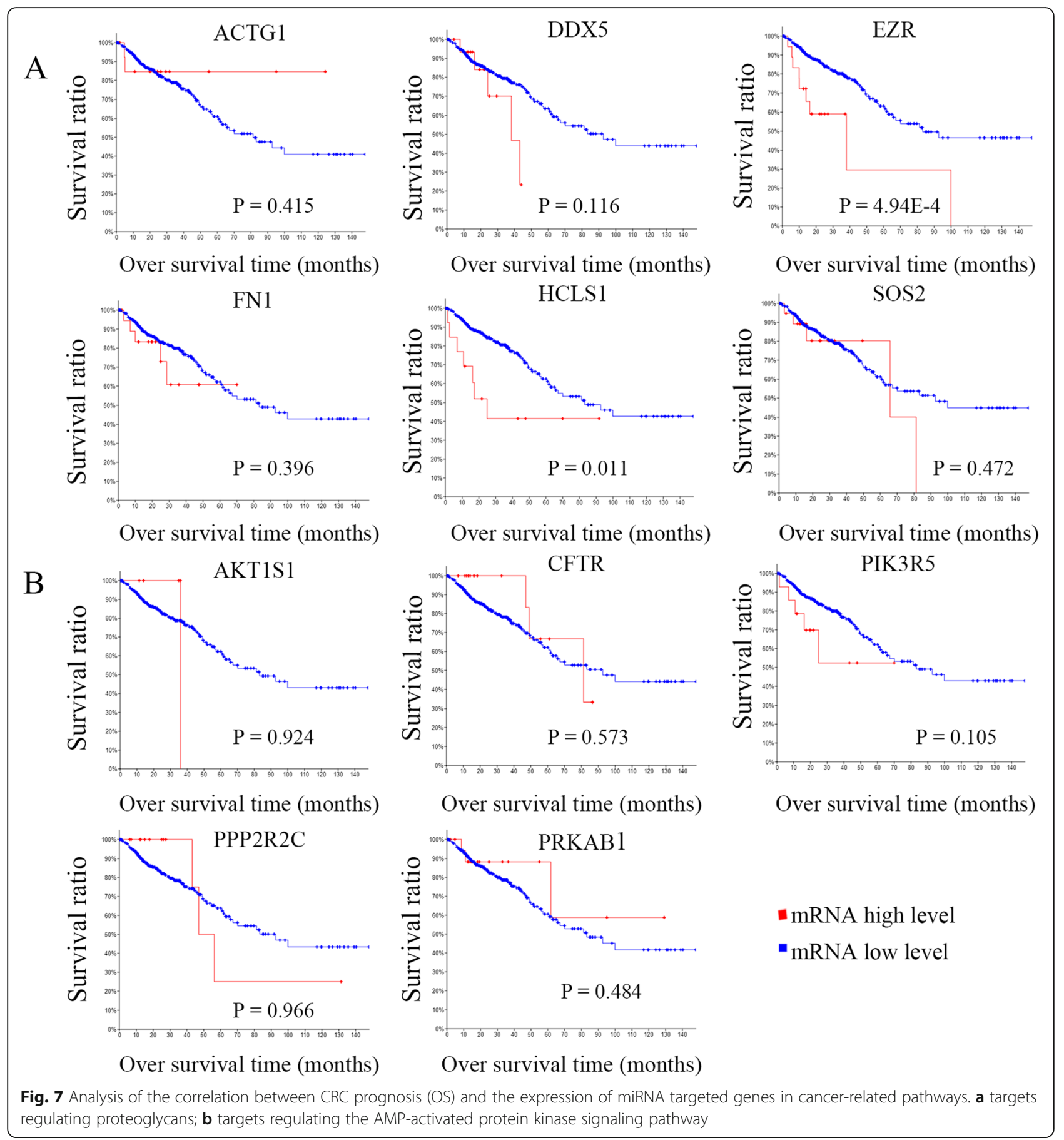

A total of 264 target genes of 85 DEmiRNAs were validated using DS. Among those, only one novel DEmiRNAs (PC-5p-23775_61), was identified to direct cleave the target the gene lysine demethylase 1 (KDM1B). KDM1B regulated histone lysine methylation and acted as an epigenetic marker regulating gene expression and chromatin function [34]. In addition, KDM1B was also reported to be targeted by $\mathrm{miR}-215$ to mediate gliomainitiating cells to adapt to hypoxia [35]. Functional analysis indicated that these potential miRNA targets were enriched in the proteoglycans in cancer and AMPK signaling pathway. Seven target genes, namely DDX5, SOS2, ACTG1, EZR, FN1, HCLS1 and PIK3R5, were involved in proteoglycans in cancer signaling pathway. mmu-mir-6236-p3_2 targeted the FN1 gene, and hsamiR-1296-5p targeted the HCLS1 gene, to respond to the MAPK signaling pathway. Another miRNA, hsamiR-665_R-2, was involved in proliferation and survival, 


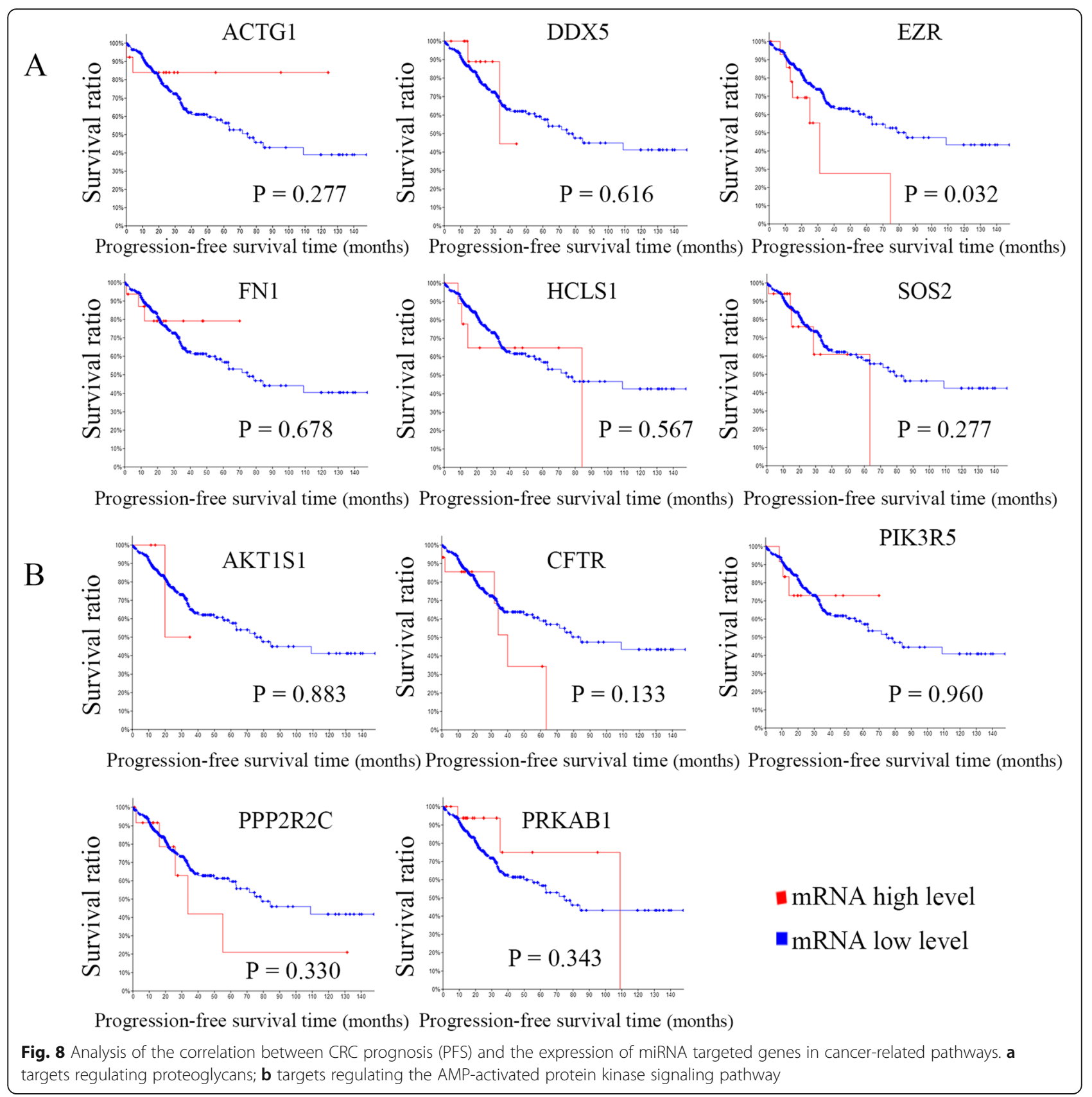

and regulated the expression of the PIK3R5 protein in the PI3K-Akt signaling pathway. The expression of these miRNA targets was validated by qPCR, which was consistent with high-throughput sequencing data (Fig. 5). Moreover, five target genes, namely hsa-miR-1268a-mediated cleavage of AKT1S1, hsa-miR-383-5p-mediated cleavage of CFTR, hsa-miR-665_R-2-mediated cleavage of PIK3R5, hsa-miR-548ae-5p-mediated cleavage of PRKAB1, and hsa-mir-7110-p3_1ss18AC-mediated cleavage of PPP2R2C, were involved in the AMPK signaling pathway. These findings indicated that the vast majority of miRNAs functioned at different manners to regulate CRC by guiding the cleavage of target genes, which attracted us to explore the clinical signification of these miRNA targets.

Since the number of patient samples was limited, we interrogated the CRC dataset of TCGA at cBioPortal to investigate the relationship between miRNA targets in cancer-related signal pathways and clinical features. After systemic analysis of prognosis-related miRNA targets in cancer-related signal pathways, interestingly, we found that EZR and HCLS1 had the potential prognostic characteristics with CRC regarding OS or recurrence, which drove us to further investigate the correlation 
A
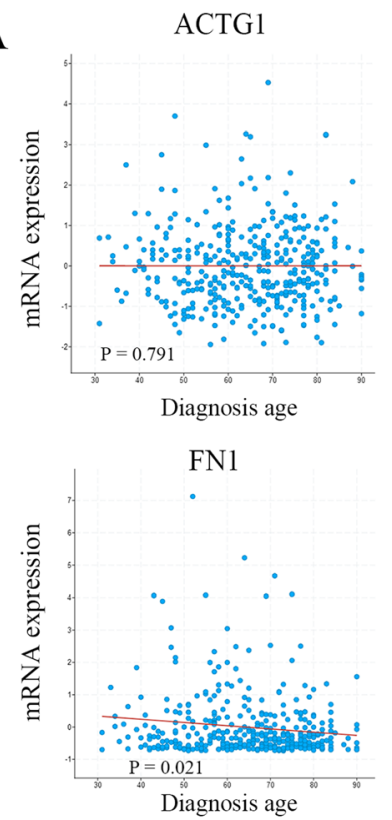

B
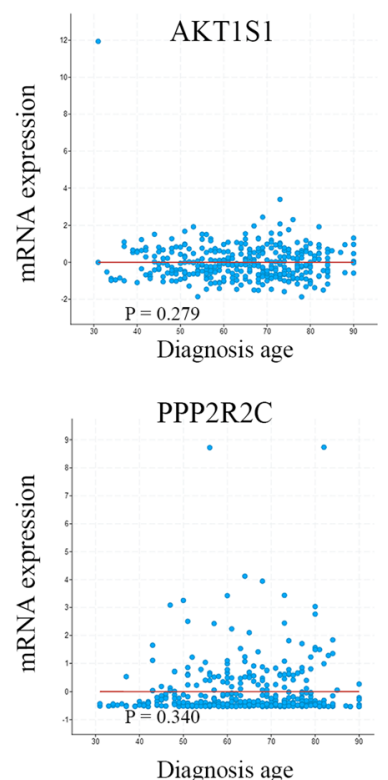

DDX5
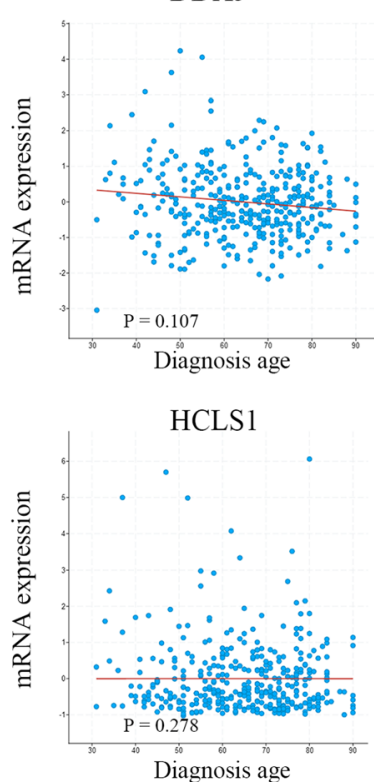

CFTR
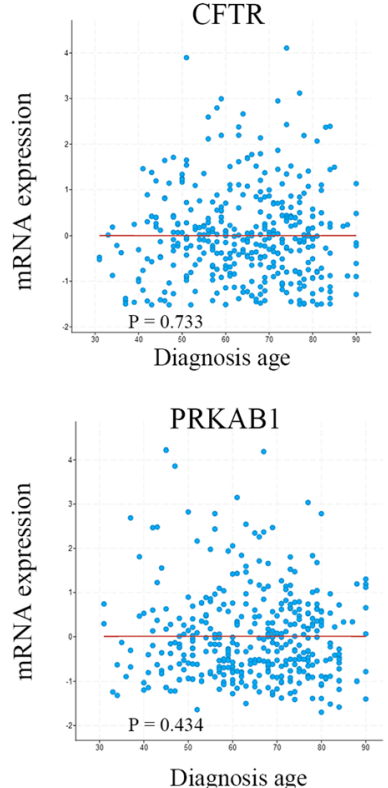

EZR

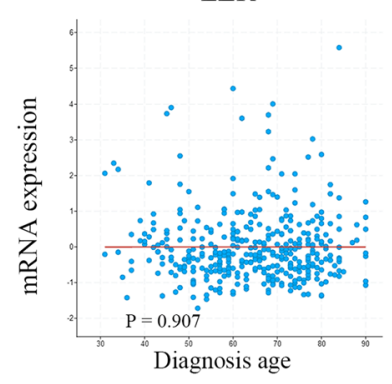

SOS2

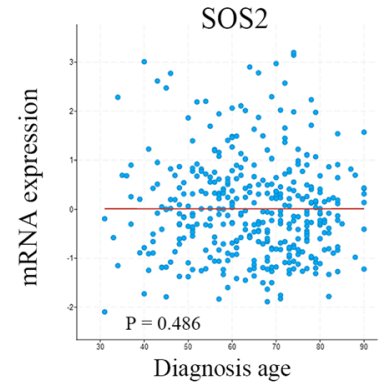

PIK3R5

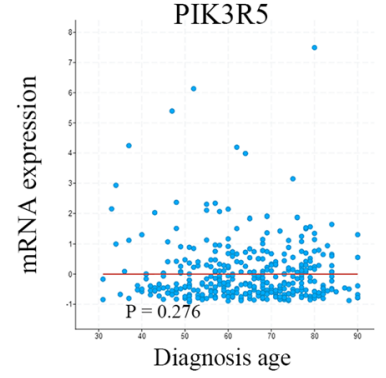

Fig. 9 Analysis of the correlation between CRC diagnosis age and the expression of miRNA targeted genes in cancer-related pathways. a targets regulating proteoglycans; $\mathbf{b}$ targets regulating the AMP-activated protein kinase signaling pathway

between them and other clinical features. However, apart from FN1 being identified to be associated with diagnosis age, other clinical features, including sex, tumor stage, histologic type, metastatic status and KRAS mutation, were evaluated separately, and no significant associations with 11 miRNA targets were observed.

\section{Conclusion}

In this study, a systematic and comprehensive evaluation of CRC-related miRNAs and their endonucleolytic cleaved target genes was conducted through high- throughput sequencing and DS. The present findings support previous evidence showing that mRNA cleavage guided by miRNAs occurs in animal. Furthermore, some miRNAs may have the potential as prognosis markers of CRC. These findings promoted and improved the better appreciation of the novel interaction mode between miRNAs and target genes in CRC .

\section{Supplementary information}

Supplementary information accompanies this paper at https://doi.org/10. 1186/s12885-020-06717-4. 
Additional file 1: Table S1. Clinical characteristics of the study population. Table S2. The primers of miRNAs validated by qRT-PCR. Table S3. Annotation of sRNAs sequences. Table S4. All Expressed known miRNA. Table S5. All novel or predicted candidate miRNAs (PC miRNAs) in this study. Table S6. Differentially expressed known miRNAs between the $C T$ and $C N$ libraries. Table S7. Differentially expressed novel miRNAs between the $\mathrm{CT}$ and $\mathrm{CN}$ libraries. Table S8. Differentially expressed miRNAs between the early-stage I-II (CT-E) and advanced-stage III-IV (CT-A) libraries. Table S9. All targets of known miRNAs. Table S10. All targets of novel miRNAs. Table S11. Potential target genes of DEmiRNAs.

Additional file 2: Figure S1. The distribution of the lengths of miRNAs in $\mathrm{CT}$ and $\mathrm{CN}$ libraries.

Additional file 3: Figure S2. Analysis of the correlation between CRC tumor stage and the expression of miRNA targeted genes in cancerrelated pathways. (A) targets regulating proteoglycans; (B) targets regulating the AMP-activated protein kinase signaling pathway.

Additional file 4: Figure S3. Analysis of the correlation between CRC histologic type and the expression of miRNA targeted genes in cancerrelated pathways. (A) targets regulating proteoglycans; (B) targets regulating the AMP-activated protein kinase signaling pathway.

Additional file 5: Figure S4. Analysis of the correlation between CRC KRAS mutation and the expression of miRNA targeted genes in cancerrelated pathways. (A) targets regulating proteoglycans; (B) targets regulating the AMP-activated protein kinase signaling pathway.

\section{Abbreviations}

CRC: colorectal cancer; miRNAs: microRNAs; sRNA: small RNA; DS: degradome sequencing; GO: gene ontology; KEGG: Kyoto Encyclopedia of Genes and Genomes; DEmiRNAs: differentially expressed miRNAs; RT-PCR: reverse transcription-polymerase chain reaction; DAVID: Database for Annotation, Visualization and Integrated Discovery; TPM: transcripts per million; NCBI: National Center for Biotechnology Information; BP: biological process; MF: molecular function; CC: cellular component; mESCs: mouse embryonic stem cells; MVP: major vault protein; AKT3: AKT serine/threonine kinase 3; KDM1B: lysine demethylase 1; DDX5: DEAD-box helicase 5; SOS2: SOS Ras/ Rho guanine nucleotide exchange factor 2; ACTG1: actin gamma 1; EZR: ezrin; FN1: fibronectin 1; HCLS1: hematopoietic cell-specific Lyn substrate 1; PIK3R5: phosphoinositide-3-kinase regulatory subunit 5; AKT1S1: AKT1 substrate 1; CFTR: cystic fibrosis transmembrane conductance regulator; PRKAB1: protein kinase AMP-activated non-catalytic subunit beta 1 ; PPP2R2C: rotein phosphatase 2 regulatory subunit B gamma; SNPs: single nucleotide polymorphisms

\section{Acknowledgements}

Not applicable.

\section{Authors' contributions}

$F Z, W T$ and $Y D$ conceived and designed the experiments. FZ, DT, YX, HH and YW performed the experiments. FZ and JD analyzed the data. FZ and LL collected the samples. FZ drafted the manuscript. All authors read and approved the final manuscript.

\section{Funding}

This work was supported by the National Natural Science Foundation of China (Grant No. 81671596); Science and Technology Planning Project of Guangdong Province, China (No.2016A050503009); Science and Technology Planning Project of Shenzhen Municipality, China (No. JCYJ20170307095037263, JCYJ20160422164313440); Chinese Postdoctoral Science Foundation (2017 M612849) and Science and Technology Planning Project of Guangdong Province, China (No. 2016A020215027) in China. The funding bodies had no roles in the design of the study and collection, analysis, and interpretation of data and in writing the manuscript.

\section{Availability of data and materials}

The datasets generated and/or analyzed during the current study are available in the GEO repository, accession no. GSE125961 and GSE125962.

\section{Ethics approval and consent to participate}

All procedures performed in studies involving human participants were in accordance with the ethical standards of the Ethical Committee and Study Protocols Review Boards of Jinan University (Guangzhou, China) and with the 1964 Helsinki declaration and its later amendments or comparable ethical standards. All participants received information on the aim and procedures of the study, and written informed consent was obtained from all subjects.

\section{Consent for publication}

Not applicable.

\section{Competing interests}

The authors declare that they have no competing interests.

\section{Author details}

${ }^{1}$ Clinical Medical Research Center, The Second Clinical Medical College of Jinan University (Shenzhen People's Hospital), 1017 North Rd Dongmen, Luohu District, Shenzhen, China. ${ }^{2}$ Integrated Chinese and Western Medicine Postdoctoral research station, Jinan University, Guangzhou, China.

${ }^{3}$ Department of Pathophysiology, Key Laboratory of the State Administration of Traditional Chinese Medicine, Medical College of Jinan University, Guangzhou, China. ${ }^{4}$ Department of Oncology, Shenzhen Hospital of Southern Medical University, Shenzhen, China.

Received: 18 September 2019 Accepted: 5 March 2020

Published online: 23 March 2020

\section{References}

1. Lee RC, Feinbaum RL, Ambros V. The C. elegans heterochronic gene lin-4 encodes small RNAs with antisense complementarity to lin-14. Cell. 1993;75(5):843-54.

2. Jones-Rhoades MW, Bartel DP, Bartel B. MicroRNAS and their regulatory roles in plants. Annu Rev Plant Biol. 2006:57:19-53.

3. Wang Y, Li L, Tang S, Liu J, Zhang H, Zhi H, Jia G, Diao X. Combined small RNA and degradome sequencing to identify miRNAs and their targets in response to drought in foxtail millet. BMC Genet. 2016;17:57.

4. Candar-Cakir B, Arican E, Zhang B. Small RNA and degradome deep sequencing reveals drought-and tissue-specific micrornas and their important roles in drought-sensitive and drought-tolerant tomato genotypes. Plant Biotechnol J. 2016;14(8):1727-46.

5. Ma X, Zhang X, Zhao K, Li F, Li K, Ning L, He J, Xin Z, Yin D. Small RNA and Degradome Deep Sequencing Reveals the Roles of microRNAs in Seed Expansion in Peanut (Arachis hypogaea L.). Front Plant Sci. 2018;9:349.

6. Bartel DP. MicroRNAs: target recognition and regulatory functions. Cell. 2009;136(2):215-33.

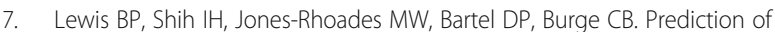
mammalian microRNA targets. Cell. 2003;115(7):787-98.

8. Jain S, Rana V, Tridibes A, Sunil S, Bhatnagar RK. Dynamic expression of miRNAs across immature and adult stages of the malaria mosquito Anopheles stephensi. Parasit Vectors. 2015:8:179.

9. Bracken CP, Szubert JM, Mercer TR, Dinger ME, Thomson DW, Mattick JS, Michael MZ, Goodall GJ. Global analysis of the mammalian RNA degradome reveals widespread miRNA-dependent and miRNA-independent endonucleolytic cleavage. Nucleic Acids Res. 2011;39(13):5658-68.

10. Karginov FV, Cheloufi S, Chong MM, Stark A, Smith AD, Hannon GJ. Diverse endonucleolytic cleavage sites in the mammalian transcriptome depend upon microRNAs, Drosha, and additional nucleases. Mol Cell. 2010;38(6):781-8.

11. Yekta S, Shih $\mathrm{H}$, Bartel DP. MicroRNA-directed cleavage of HOXB8 mRNA. Science. 2004;304(5670):594-6.

12. Park JH, Ahn S, Kim S, Lee J, Nam JW, Shin C. Degradome sequencing reveals an endogenous microRNA target in C. elegans. FEBS Lett. 2013; 587(7):964-9.

13. Cass AA, Bahn JH, Lee JH, Greer C, Lin X, Kim Y, Hsiao YH, Xiao X. Global analyses of endonucleolytic cleavage in mammals reveal expanded repertoires of cleavage-inducing small RNAs and their targets. Nucleic Acids Res. 2016;44(7):3253-63.

14. Abend JR, Uldrick T, Ziegelbauer JM. Regulation of tumor necrosis factor-like weak inducer of apoptosis receptor protein (TWEAKR) expression by Kaposi's sarcoma-associated herpesvirus microRNA prevents TWEAK-induced apoptosis and inflammatory cytokine expression. J Virol. 2010;84(23):12139-51. 
15. Gottwein E, Corcoran DL, Mukherjee N, Skalsky RL, Hafner M, Nusbaum JD, Shamulailatpam P, Love CL, Dave SS, Tuschl T, et al. Viral microRNA targetome of KSHV-infected primary effusion lymphoma cell lines. Cell Host Microbe. 2011;10(5):515-26.

16. German MA, Pillay M, Jeong DH, Hetawal A, Luo S, Janardhanan P, Kannan $\checkmark$, Rymarquis LA, Nobuta K, German R, et al. Global identification of microRNA-target RNA pairs by parallel analysis of RNA ends. Nat Biotechnol. 2008:26(8):941-6.

17. World Health Organization. World Cancer Report 2014. (2014) Available at: http:// www.who.int/cancer/publications/WRC_2014/en/ (Accessed 14 Feb 2019).

18. Muhammad S, Kaur K, Huang R, Zhang Q, Kaur P, Yazdani HO, Bilal MU, Zheng J, Zheng L, Wang XS. MicroRNAs in colorectal cancer: role in metastasis and clinical perspectives. World J Gastroenterol. 2014;20(45): 17011-9.

19. Mohammadi A, Mansoori B, Baradaran B. The role of microRNAs in colorectal cancer. Biomed Pharmacotherapy. 2016;84:705-13.

20. Schetter AJ, Okayama H, Harris CC. The role of microRNAs in colorectal cancer. Cancer J. 2012;18(3):244-52.

21. Ambros V, Bartel B, Bartel DP, Burge CB, Carrington JC, Chen X, Dreyfuss G, Eddy SR, Griffiths-Jones S, Marshall M, et al. A uniform system for microRNA annotation. Rna. 2003:9(3):277-9.

22. Addo-Quaye C, Miller W, Axtell MJ. CleaveLand: a pipeline for using degradome data to find cleaved small RNA targets. Bioinformatics. 2009; 25(1):130-1.

23. Sun $X$, Zhai $H$, Chen $X$, Kong R, Zhang X. MicroRNA-1271 suppresses the proliferation and invasion of colorectal cancer cells by regulating metadherin/Wnt signaling. J Biochem Mol Toxicol. 2018;32(2):e22028.

24. Zheng $K$, et al. Epigenetic silencing of miR-490-3p promotes development of an aggressive colorectal cancer phenotype through activation of the Wnt/ $\beta$-catenin signaling pathway. Cancer letters. 2016;376(1):178-87.".

25. Eldem V, Celikkol Akcay U, Ozhuner E, Bakir Y, Uranbey S, Unver T. Genomewide identification of miRNAs responsive to drought in peach (Prunus persica) by high-throughput deep sequencing. PLoS One. 2012;7(12):e50298.

26. Koga $Y$, Yasunaga M, Takahashi A, Kuroda J, Moriya Y, Akasu T, Fujita S, Yamamoto S, Baba H, Matsumura Y. MicroRNA expression profiling of exfoliated colonocytes isolated from feces for colorectal cancer screening. Cancer Prev Res. 2010;3(11):1435-42.

27. Wu X, Somlo G, Yu Y, Palomares MR, Li AX, Zhou W, Chow A, Yen Y, Rossi $J$ J, Gao $H$, et al. De novo sequencing of circulating miRNAs identifies novel markers predicting clinical outcome of locally advanced breast cancer. J Transl Med. 2012;10:42.

28. Wang CJ, Zhou ZG, Wang L, Yang L, Zhou B, Gu J, Chen HY, Sun XF. Clinicopathological significance of microRNA-31, -143 and -145 expression in colorectal cancer. Dis Markers. 2009;26(1):27-34

29. Yamada Y, Sakuma J, Takeuchi I, Yasukochi Y, Kato K, Oguri M, Fujimaki T, Horibe H, Muramatsu M, Sawabe M, et al. Identification of rs7350481 at chromosome 11q23.3 as a novel susceptibility locus for metabolic syndrome in Japanese individuals by an exome-wide association study. Oncotarget. 2017:8(24):39296-308.

30. Xiao YS, Zeng, Liang YK, Wu Y, Li MF, Qi YZ, Wei XL, Huang WH, Chen M, Zhang GJ. Major vault protein is a direct target of Notch1 signaling and contributes to chemoresistance in triple-negative breast cancer cells. Cancer Lett. 2019:440-441:156-67.

31. Sui GQ, Fei D, Guo F, Zhen X, Luo Q, Yin S, Wang H. MicroRNA-338-3p inhibits thyroid cancer progression through targeting AKT3. Am J Cancer Res. 2017;7(5):1177-87

32. Wang YX, Zhu HF, Zhang ZY, Ren F, Hu YH. MiR-384 inhibits the proliferation of colorectal cancer by targeting AKT3. Cancer Cell Int. 2018;18:124.

33. Guo T, Sakai A, Afsari B, Considine M, Danilova L, Favorov AV, Yegnasubramanian S, Kelley DZ, Flam E, Ha PK, et al. A novel functional splice variant of AKT3 defined by analysis of alternative splice expression in HPV-positive Oropharyngeal cancers. Cancer Res. 2017;77(19):5248-58.

34. Karytinos A, Forneris F, Profumo A, Ciossani G, Battaglioli E, Binda C, Mattevi A. A novel mammalian flavin-dependent histone demethylase. J Biol Chem. 2009;284(26):17775-82.

35. Hu J, Sun T, Wang H, Chen Z, Wang S, Yuan L, Liu T, Li HR, Wang P, Feng Y, et al. MiR-215 is induced post-transcriptionally via HIF-Drosha complex and mediates Glioma-initiating cell adaptation to hypoxia by targeting KDM1B. Cancer Cell. 2016;29(1):49-60.

\section{Publisher's Note}

Springer Nature remains neutral with regard to jurisdictional claims in published maps and institutional affiliations.
Ready to submit your research? Choose BMC and benefit from:

- fast, convenient online submission

- thorough peer review by experienced researchers in your field

- rapid publication on acceptance

- support for research data, including large and complex data types

- gold Open Access which fosters wider collaboration and increased citations

- maximum visibility for your research: over $100 \mathrm{M}$ website views per year

At BMC, research is always in progress.

Learn more biomedcentral.com/submissions 